\title{
FACTORS REGULATING THE BIOSYNTHESIS OF
}

\author{
VARIOUS PROSTAGLANDINS \\ w. Lands, R. Lee and W. Smith \\ Department of Biclocical Chemistry \\ University of Michigan \\ Ann Arbor, Mich.
}

The biosynthetic pathways to various prostaglandins from polyunsaturated fatty acids have been outlined by numerous reports from the laboratories of Bergstrym(1967) and Samuelsson (1967), in Sweden, and van Dorp (1967), in The Netherlands.

Three years ago I was privileged to spend six months at the Karolinska Institute working in collaboration with Professor B. Samuelsson. At that time we were able to develop thin-layer techniques which allowed quantitative measurement of the many different products appearing during prostaglandin biosynthesis. This led to the identification of the unusual prostaglandin,11-keto-PGF $1 \alpha$, from reaction mixtures (Granstrom et al,1968), and a recognition that the oxidative cyclization most probably occurs after the liberation of the essential acid(Lands \& Samuelsson, 1968), by action of an acylhydrolase. Last year I began again to use those methods to examine in more detail the regulation of prostaglandin biosynthesis. A useful review of the factors in the biosynthesis of prostaglandins recognized up to that point was provided by Samuelsson in that year's Progress of Biochemical Pharmacology (Samuelsson, 1969).

The present report is designed to indicate briefly certain aspects of the multi-enzyme system that are still being investigated. A principal feature in our work is regarding each enzyme-catalyzed step as a potentially independent process to be examined individually. Thus, prostaglandins are formed from precursor lipids by many sequential reactions, as shown in Fig. 1 .

The release of the unesterified acid from esterified forms seems necessary for any appreciable synthesis to occur (Lands \& Samuelsson, 1968). At the present time the recognized acylhydrolase activities are relatively low and not well characterized. The subsequent oxidative and rearrangement steps are the main concern of this report. They were followed in our experiments by incubating radioactive arachidonate with tissue preparations.

The various products of the different reactions were extracted from the reaction mixtures and separated from each other by TLC on silica gel G. After developing to $15 \mathrm{~cm}$ in benzene:dioxane:acetic acid:formic acid (82:14:1:1), the plate was dried briefly and redeveloped to $7.5 \mathrm{~cm}$ in acetone:methylene chloride $(60: 40)$ to get better separation of the products more polar than monohydroxy acids. With these 


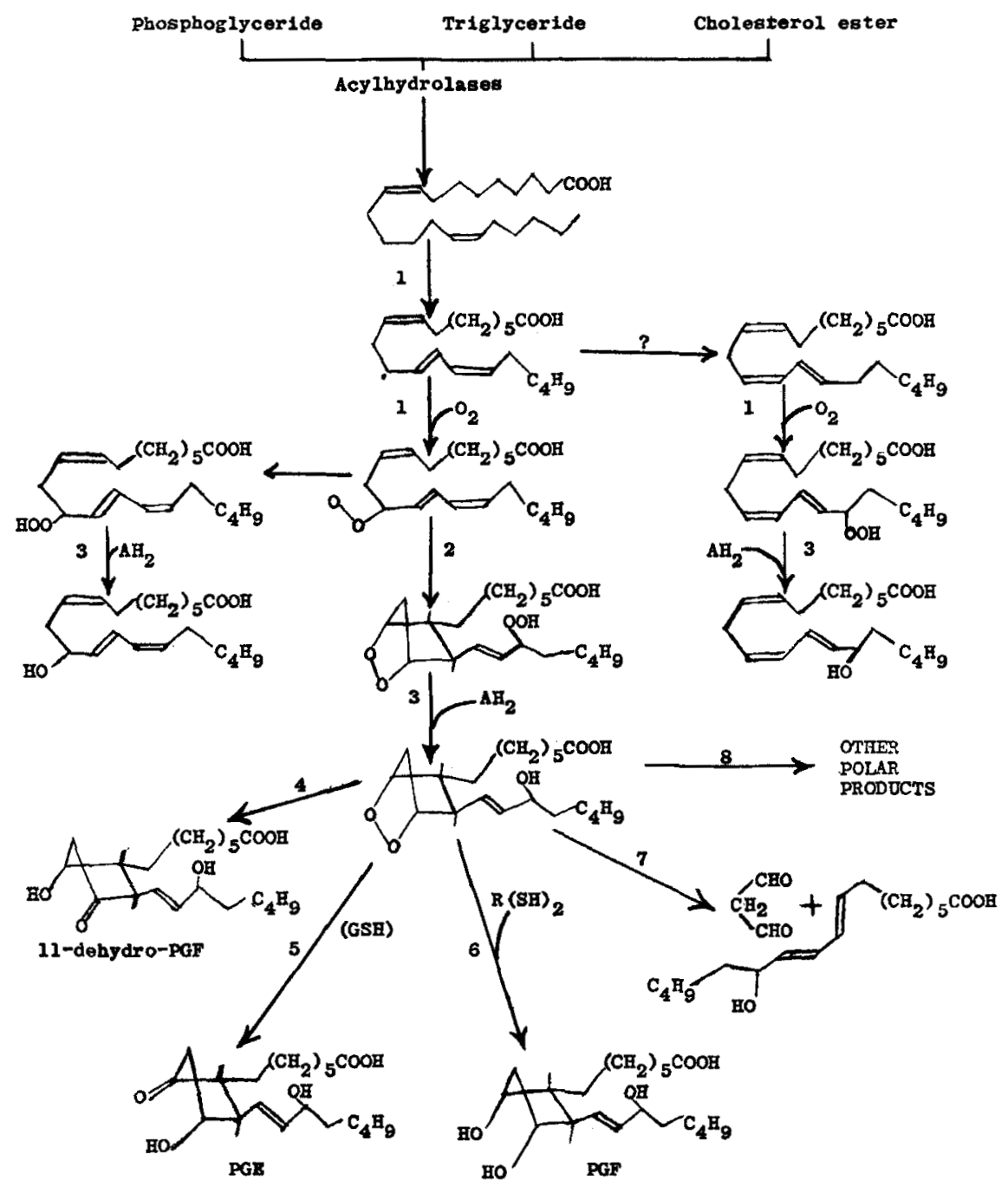

Figure 1. Biosynthetic routes to various prostaglandins. solvents there was no separation of the products derived from arachidonate (e.g., $\mathrm{PGE}_{2}$ ) from the corresponding products from eicosatrienoate $\left(\mathrm{PGE}_{1}\right)$. The silicic acid was scraped from regions of the plate, as indicated in Fig.2, and the radioactivity determined by liquid scintillation spectometry. The non-incubated control (left side of Fig.2) shows the recovery of unreacted substrate at an $R_{F}$ value of 0.9 . The radioactive materials in the 6 -min incubation mixture were principally unreacted acid and PGE. The changes in 
(A)

0. Control

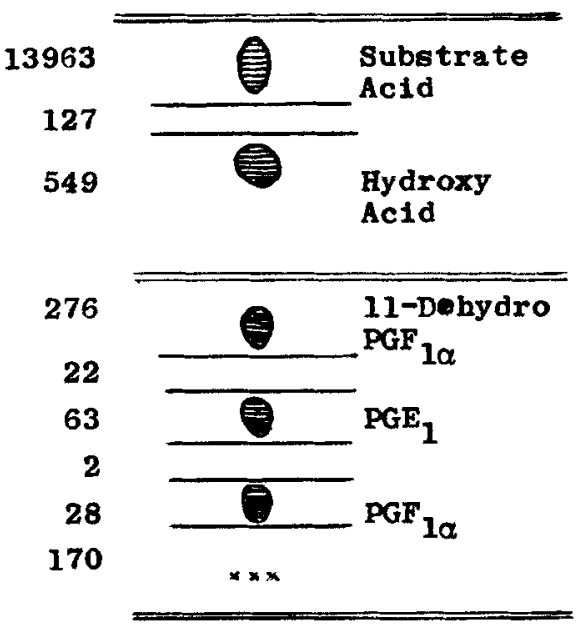

(B)

6' Rraction

$(B-A)$

8984

[-4979]

71

-56 ]

1393

844

1048

772

200

178

2611

2548

431

429

189

161

292

Figure 2. Thin-layer chromatographic separation of reaction products.

radioisotope produced upon incubation are shown in the last column at the right. The decrease in free acid from the top of the plate is reflected in corresponding increases in the hydroxy acid and more polar regions of the chromatogram. This TLC technique was used to follow, insofar as possible, the various enzyme-catalyzed steps related to prostaglandin production. By selecting early time points we attempted to obtain values reflecting initial velocities and enzymic activities, rather than the amounts of product that might accumulate with time.

\section{The formation of PGE compounds}

An example of the application of the TLC techniques is illustrated by the effect of glutathione on the overall synthetic paths for prostaglandin production. We wished to know if the greater amount of PGE produced in the presence of glutathione(Samuelsson, 1967; van Dorp, 1967; Lapidus et al, 1968) is in addition to, or at the expense of, the other products observed. The former would suggest a stimulation of overall oxygenation and cyclization reaction $(1,2,3$ and 5 in Fig.1), whereas the latter would indicate a specific stimulation of the breakdown of cyclic peroxide to PGE(reaction 5). Since the C17 and C20 hydroxy acids originate by different routes, the hydroxy acid region of the thin layers was scraped, the acids eluted, derivatized, separated by GLC and their radioactivity determined separately. In this way, the amount of hydroxyheptadecadienoate could be included with 
that of other products arising after the cyclization reaction.

Results from two experiments with homogenates of sheep vesicular glands are shown in Table 1. As the molar ratio of

Table 1. Effect of glutathione on prostaglandin formation.

\begin{tabular}{|c|c|c|c|c|c|c|}
\hline \multirow{2}{*}{$\begin{array}{c}\text { Cofactor } \\
\text { Ratio } \\
\text { (GSH/Acid) }\end{array}$} & \multicolumn{5}{|c|}{ Products* } & \multirow[b]{2}{*}{ PGE } \\
\hline & $\left(2 \frac{\mathrm{a}}{\bar{O} \mathrm{c}}\right)$ & $\frac{b}{(17 c)}$ & C & $\frac{d}{\text { (PGF) }}$ & $(b+c+d)$ & \\
\hline $\begin{array}{r}0 \\
1 \\
10 \\
0 \\
10 \\
30\end{array}$ & $\begin{array}{r}.4 \\
.4 \\
.8 \\
2.3 \\
.9 \\
1.5\end{array}$ & $\begin{array}{r}.6 \\
.3 \\
.5 \\
2.3 \\
.2 \\
.4\end{array}$ & $\begin{array}{l}2.7 \\
2.1 \\
2.0 \\
8.0 \\
2.1 \\
3.0\end{array}$ & $\begin{array}{r}.9 \\
.8 \\
.4 \\
2.8 \\
.5 \\
.7\end{array}$ & $\begin{array}{r}4.2 \\
3.2 \\
2.9 \\
13.1 \\
2.8 \\
4.1 \\
\end{array}$ & $\begin{array}{r}1.5 \\
2.4 \\
3.9 \\
3.3 \\
13.5 \\
11.5 \\
\end{array}$ \\
\hline
\end{tabular}

*the products listed under $a$ and $b$ are acyclic hydroxy acids(C17 and C20, respectively). Column $c$ indicates the amount in the 1l-dehydro-PGF region and $d$, that in the PGF region. All values are as nmoles.

glutathione to substrate acid was increased, the rate of PGE production also rose. However, the production of radioactive products in regions $b, c$ and $d, a l l$ regarded to be derived from the cyclic endoperoxide, decreased, while that of PGE increased. Since all the products mentioned would require reduction of the 15-hydroperoxide, the reduced glutathione does not seem to act principally by stimulating Type 3 reductions to an alcohol. Rather, the decreased production by routes 4,6 and 7 suggest that glutathione is selectively accelerating the rearrangement reaction 5 .

Studies with highly purified systems are needed to be certain whether the glutathione is specifically serving in reaction 5 as a nucleophilic cofactor without oxidation or reduction. Such a reaction could occur in a manner analogous to the well established role of glutathione in glyoxalase action(Lohmann, 1932; Racker, 1951; Rose, 1957) (see Fig.3). The conversion of methylglyoxal to lactate is catalyzed by two protein fractions, glyoxalase I and glyoxalase II. The first enzyme requires glutathione to catalyze an internal 1 , 2 hydride shift and forms the lactyl thiolester. The second

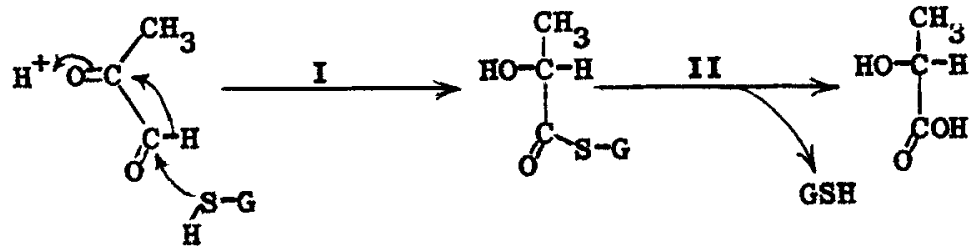

Figure 3. The role of glutathione in glyoxalase action. An enzyme-catalyzed glutathione specific internal 1 , 2 hydride shift. 
enzyme, an acyl hydrolase, liberates lactate and unaltered glutathione which can continue to serve as a cofactor without net consumption. The glutathione-assisted shift of a hydride to an adjacent incipient carbonium atom may be related to a glutathione-assisted shift of a hydride to an adjacent incipient oxonium atom, as illustrated in Fig. 4.

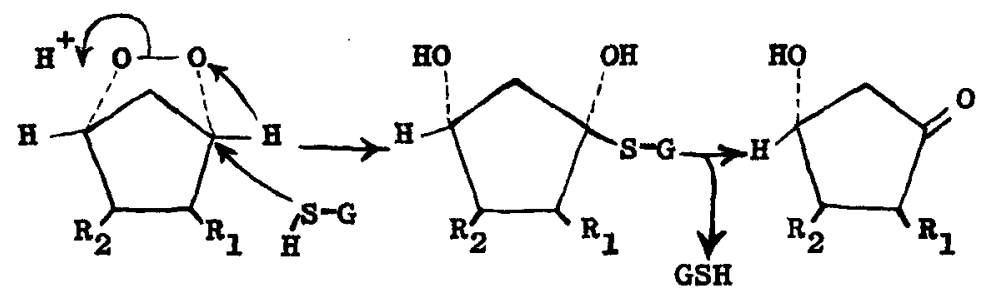

Figure 4. Proposed mechanism for a glutathione assisted 1, 2 hydride shift in PGE formation.

In the latter case, however, the resultant intermediate is a metastable thiohemiketal(rather than a stable, isolable thiolester) so that proof for its existence would be harder to obtain. A corresponding 1,2 shift to a positive oxygen atom occurs with a phenyl group during the acid-catalyzed decomposition of cumene hydroperoxide(Seubold \& Vaughan, 1953), as shown in Fig.5. In principle, a hydride shift could

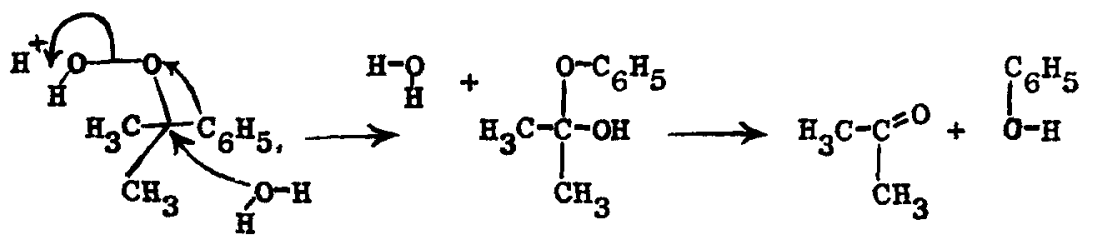

Figure 5. Rearrangement of cumene hydroperoxide.

occur in analogous fashion, with all lipid peroxides, when no phenyl groups are available for migration to the incipient oxonium. A nucleophile would then combine with these intermediate carbon ions in the same way GSH is proposed to react with the rearranged intermediate to form PGE (Fig.4)

An interesting variation of this reaction mechanism may prevail under conditions which produce 11-dehydro-PGF (Granström et al, 1968). When reduced glutathione has been removed from the enzyme complex the cyclic endoperoxide may be attacked by a nucleophile of the enzymic protein to give migration of the ll-hydride rather than the normally preferred 9-hydride. In some incubations we were able to obtain $60 \%$ of the radioactive product chromatographing in the 11-dehydro-PGF region. 
Most reactions of the cyclic peroxide intermediate are non-oxidative rearrangements and do not require additional cofactors. A variety of other oxygenated cyclic acids, including PGB (Green \& Samuelsson, 1964) and 8-iso-PGE (Daniels et al, 1968) have chromatographic properties similar to ll-dehydro-PGF. In addition, the metal catalyzed decomposition of hydroperoxide produces highly polar material (o'Brien, 1969) that also may chromatograph with ll-dehydro-PGF. Therefore, this region of the plate cannot be unequivocally assigned to that particular product.

Formation of PGF compounds

One reaction of the cyclic peroxide that is of major interest to us is designated \#6 in Fig.l. This reaction differs from the others in requiring an additional source of electrons to reductively cleave the endoperoxide to a diol. our first attempts at characterizing the separate catalysts and required cofactors were directed at separating the subcellular membrane fractions to see whether one fraction produced more PGF relative to PGE. The principal synthetic activity sedimented from $0.25 \mathrm{M}$ sucrose homogenates in the centrifugal range of $5,000 \mathrm{~g}$ for $5 \mathrm{~min}$ to 25,000 for $30 \mathrm{~min}$. Generally, the activity was distributed between the succinate dehydrogenase, a mitochondrial marker and acyl-GPC:acyl COA acyltransferase, a microsomal marker (Eibl et al,1969). As the fractions were separated, the need for added cofactors became more apparent. In all fractions studied, the catalysts for reactions 5 and 6(see Fig.1) appeared in similar proportions, with no apparent enrichment of the reductive enzyme(\#6) in any particular fraction. Since an added source of electrons is needed for PGF production with the washed particles, a number of cofactors were considered. The following concepts led to dramatic experimental results.

We speculated that $\mathrm{Cu}^{++}$might stabilize a diradical form of the endoperoxide and assist the reductive cleavage in favor of other rearrangements. Additionally, an early choice of electron donors was the dithiol class of compounds known to form stable chelates with cut+. This combination of cofactors, more than any others tested, proved capable of shifting the production of prostaglandin derivatives in favor of PGF. Although a more detailed report will appear elsewhere, some effects of copper and dithiols are shown in Table 2. Increasing the equimolar concentration(with dithiothreitol) from 0 to $10 \mathrm{mM}$ shifted the relative amount of PGF from 0.3 to 3.2 times the amount of PGE. We observed that 1 , 3 dithioglycerol was consistently more effective than 1,2 isomer and dihydrolipoamide was the most effective of 12 dithiols tested. When added alone, the dithiols were potent inhibitors of the initial fatty acid oxidation(reaction l,Fig. 1) and high levels of dithiol, even in the presence of $\mathrm{Cu}^{++}$ diminished the total amount of oxidized products. The latter conditions, however, did allow production of PGF in large excess over PGE. The method of detecting all products and unreacted substrate on one chromatogram was particularly helpful in interpreting the decrease in overall oxidized products with the concomitant shift in ratios of products. The results in Table 3 show that when glutathione was 
Table 2. Effect of dithiols on prostaglandin F/E ratios.

\begin{tabular}{|c|c|c|c|c|}
\hline Exp. & Cofactors & $\begin{array}{c}E \\
\text { nmoles }\end{array}$ & $\begin{array}{c}F \\
\text { nmoles }\end{array}$ & $F / E$ \\
\hline 1 & $\frac{\mathrm{Cu}++ \text { + DTT }}{\text { none }}$ & $\begin{array}{l}1.7 \\
1.9 \\
1.3 \\
1.0\end{array}$ & $\begin{array}{r}.6 \\
1.5 \\
3.4 \\
3.2\end{array}$ & $\begin{array}{l}.3 \\
0.8 \\
2.6 \\
3.2\end{array}$ \\
\hline 2 & $\begin{array}{l}\mathrm{Cu}+++1,2-\mathrm{DTG} \\
\mathrm{Cu}+++1,3-\mathrm{DTG} \\
\mathrm{Cu}++ \text { DHLA }\end{array}$ & $\begin{array}{l}.23 \\
.33 \\
.28\end{array}$ & $\begin{array}{r}.42 \\
.90 \\
1.28\end{array}$ & $\begin{array}{l}1.8 \\
2.7 \\
4.6\end{array}$ \\
\hline $3 *$ & $\begin{array}{l}\mathrm{Cu}++ \\
\mathrm{Cu}+++1.2-\mathrm{DTG} \\
\mathrm{Cu}+++ \text { DHLA }\end{array}$ & $\begin{array}{r}7.7 \\
6.7 \\
10.5\end{array}$ & $\begin{array}{l}14.4 \\
16.2 \\
59.4\end{array}$ & $\begin{array}{l}1.9 \\
2.4 \\
5.7\end{array}$ \\
\hline
\end{tabular}

*experiment 3 contained 300 nmoles of substrate and was allowed to proceed for $30 \mathrm{~min}$.

the cofactor, the principal products, PGE and 11-dehydro-PGF, were converted by treatment with sodium borohydride to material co-chromatographing with PGF. On the other hand, the distribution of radioactive products of the copperdithiol system(principally PGF) was not appreciably altered by borohydride treatment. Apparentiy only very small amounts

Table 3. Separation of product from sheep vesicular glands.

\begin{tabular}{cccc}
$\begin{array}{c}\text { Cofactor } \\
\text { Added }\end{array}$ & $\begin{array}{c}\text { TLC } \\
\text { Region }\end{array}$ & $\begin{array}{c}\text { NaBH } \\
\text { Untreated } \\
\text { Treated }\end{array}$ \\
\hline \multirow{3}{*}{ GSH } & 11-dehydro-PGF & 4.4 & 0.4 \\
& PGE & 20.8 & 1.9 \\
& PGF & 1.6 & 23.3 \\
Cu++/DHLA & 11-dehydro-PGF & 0.8 & 1.2 \\
& PGE & 3.9 & 3.2 \\
& PGF & 18.2 & 19.8 \\
\hline
\end{tabular}

of PGE are produced in the presence of the dithiol cofactor. This result also helps emphasize that other polar rearrangement products may occur in regions of the TLC plate usually assigned to 11-dehydro-PGF and PGE. To further characterize the PGF produced, the trimethylsilyl ethers of the methyl esters were chromatographed on $\mathrm{SE}-30$ and $\mathrm{OV}-1$ liquid phases on GLC. The PGF produced from eicosatrienoate co-chromatographed with a PGF $1_{\alpha}$ standard and that from arachidonate corresponded to $\mathrm{PGF}_{2 \alpha}^{\alpha}$. Mass spectra* of the products resembled the spectrum published earlier (Granstrom et al, 1968) and showed characteristic fragments for PGF $1 \alpha$ and $P G F 2 \alpha$ (Fig.6)

Thus, it seems clear that in the presence of copper,

*An LKB mass spectrometer in combination with gas chromatography and a computerized data handling system was used with the assistance of the staff in the laboratory of Dr. C. C. Sweeley at Michigan State University. 


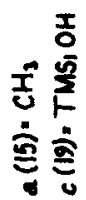

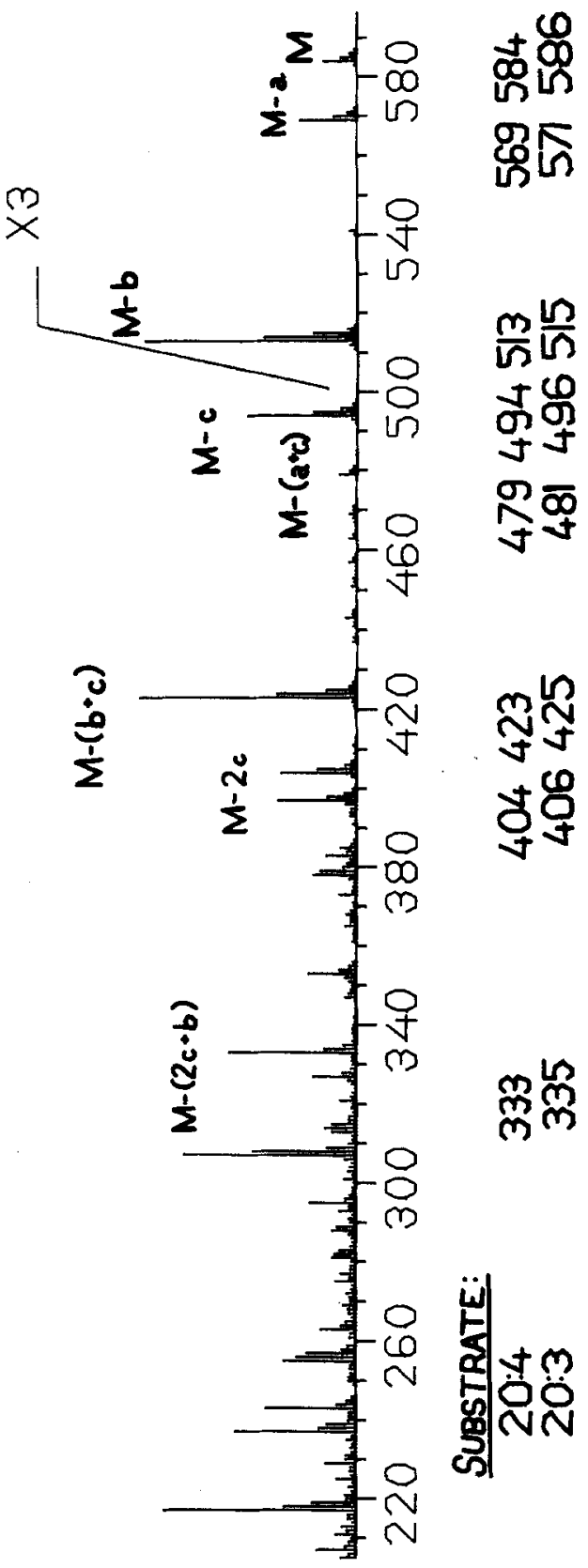




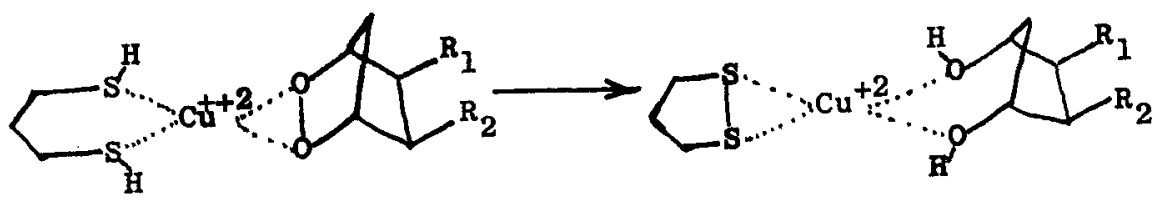

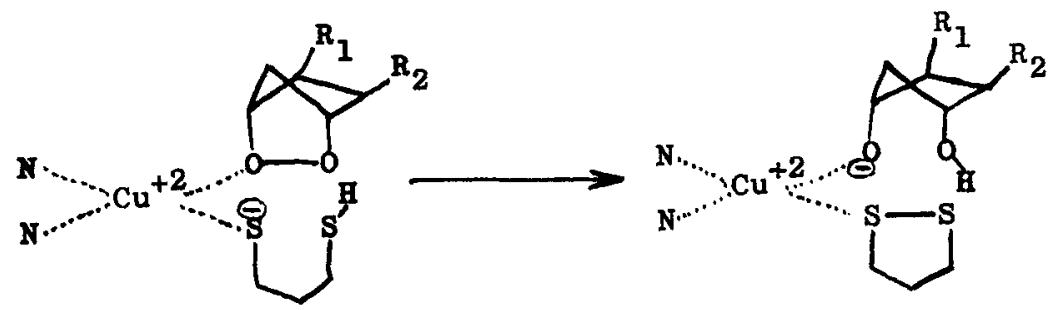

Figure 7. A role for copper in reduction of the endo-peroxperoxide to $\mathrm{PGF}$.

dihydrolipoamide can effectively prevent the formation of PGE and allow reduction of the endoperoxide to form PGF. The heuristic concept indicated in the upper part of Fig. 7 served adequately for experimental designs, but may be less favored than the mechanism shown in the lower half of the figure. The latter mechanism is somewhat analogous to that deduced by sigel for the Cut+ catalyzed decomposition of hydrogen peroxide (sigel,1969). Regardless of which mechanism is preferred on chemical considerations, both proposals need some further development to account for a physiological supply of the reductant dithiol. A likely possibility would be an enzyme dithiol which can be maintained in vitro in a reduced form by the exogenous dithiol added to our incubations.

The initial oxidation reaction

One general observation in our studies, made possible by monitoring the total products on TLC, was that incubation mixtures that were poor producers of either prostaglandin $\mathrm{E}$ or $F$ were generally poor in producing any oxidized acid from the starting substrate. It seemed as if the most labile catalysts were those involved in the initial attack by oxygen and that many incubations would have succeeded if an adequate supply of peroxide intermediates could have been assured. With this in mind we attempted to study the first reaction(Fig.l)in isolation from the many subsequent enzymic steps so that we could more systematically determine what was needed to keep it functioning. One approach had an 
unusual consequence.

We added preparations of glutathione peroxidase to incubation mixtures to artificaliy accelerate type 3 reactions and hopefully produce exclusively hydroxyeicosatetraenoic acid. Glutathione peroxidase(Little et al, 1970) catalyzes the nucleophilic cleavage of the lipid hydroperoxide, as shown in Fig.8. The enzyme isolated from rat liver catalyzes the

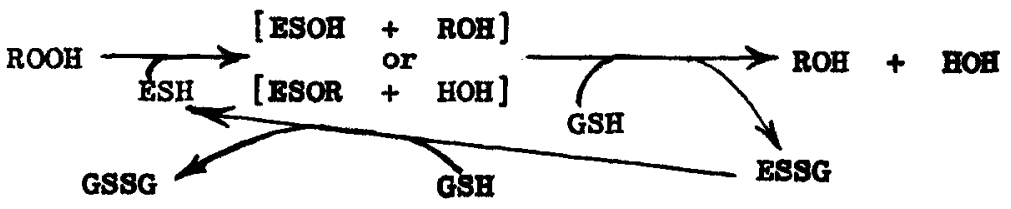

$\mathrm{ROOH}+\mathrm{GSH}+\mathrm{GSH} \longrightarrow \mathrm{GSSG}+\mathrm{ROH}+\mathrm{HOH}$

Figure 8. A mechanism for glutathione peroxidase.

reaction of a wide variety of hydroperoxides (including $\mathrm{H}_{2} \mathrm{O}_{2}$ ) with a variety of mercaptans (Little \& O'Brien, 1968). When the peroxidase was added to incubations of vesicular gland preparations it did not lead to an accumulation of hydroxyacids,but rather inhibited all oxygenation of the substrate. The inhibition was almost complete, with 765 units of peroxidase (Table 4). Since this inhibition was not caused by the glutathione peroxidase alone, and certainly not by the glutathione alone, we inferred that some active form of the vesicular gland enzyme $I$ was inactivated by the functioning glutathione peroxidase system.

Table 4. Effect of glutathione peroxidase upon prostaglandin formation by sheep vesicular gland enzymes.

\begin{tabular}{lrrrrr} 
O'Control & to u & $+35 \mathrm{u}$ & $+173 \mathrm{u}$ & $+765 \mathrm{u}$ & Product \\
\hline 13963 & 8984 & 10026 & 11893 & 13045 & \\
127 & 71 & 102 & 175 & 84 & \\
549 & 1393 & 1225 & 850 & 600 & Hydroxy acid \\
276 & 1048 & 817 & 686 & 630 & l1-dehydro-PGF \\
22 & 200 & 155 & 138 & 104 & PGE \\
63 & 2611 & 2002 & 1175 & 505 & PGF \\
2 & 431 & 300 & 241 & 67 & \\
28 & 189 & 228 & 358 & 133 & \\
170 & 292 & 334 & 260 & 374 & \\
varied amounts of peroxidase(0 to 765 units of enzyme)were \\
added to homogenates of an actone powder preparation prior \\
to the addition of the substrate, 20:4, which chromatographs \\
at the uppermost section of the thin layer plate.
\end{tabular}


To examine this phenomenon in more detail we employed soybean lipoxygenase as a model dioxygenase system for studying the initial attack by $\mathrm{O}_{2}$. Hamberg and Samuelsson (1967) have reported that an initial removal of the 13-L hydrogen is common to both lipoxygenase and the vesicular gland system, although the $\mathrm{O}_{2}$ molecule is inserted at different positions with the two systems. The results in Fig.9 show that glutathione peroxidase, in the presence of mercaptan completely inhibits the action of soybean lipoxygenase. Thus, in one more feature the mechanism of this unusual dioxygenase resembles that facilitating the production of prostaglandins in vesicular gland. The inhibitory action is dependent upon the presence of free mercaptan and is reversed by treatment with NEM, as shown in the lower half of Fig.9. The rate of formation of conjugated diene, as measured by the increasing absorbance at $234 \mathrm{~m} \mu$ was not

nmoles/min

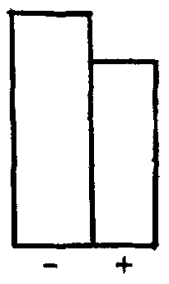

No

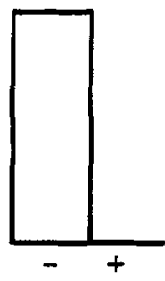

GSE

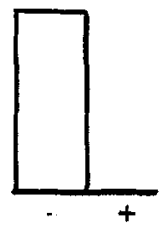

DTT

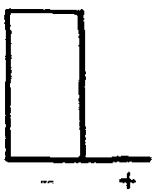

Cys

time

Figure 9. Effect of glutathione peroxidase on lipoxygenase rates. The + and - signs at the upper part of the figure indicate the presence or absence, respectively, of the peroxidase. The lower curve indicates the formation of conjugated diene in the presence of glutathione with additions as noted. 

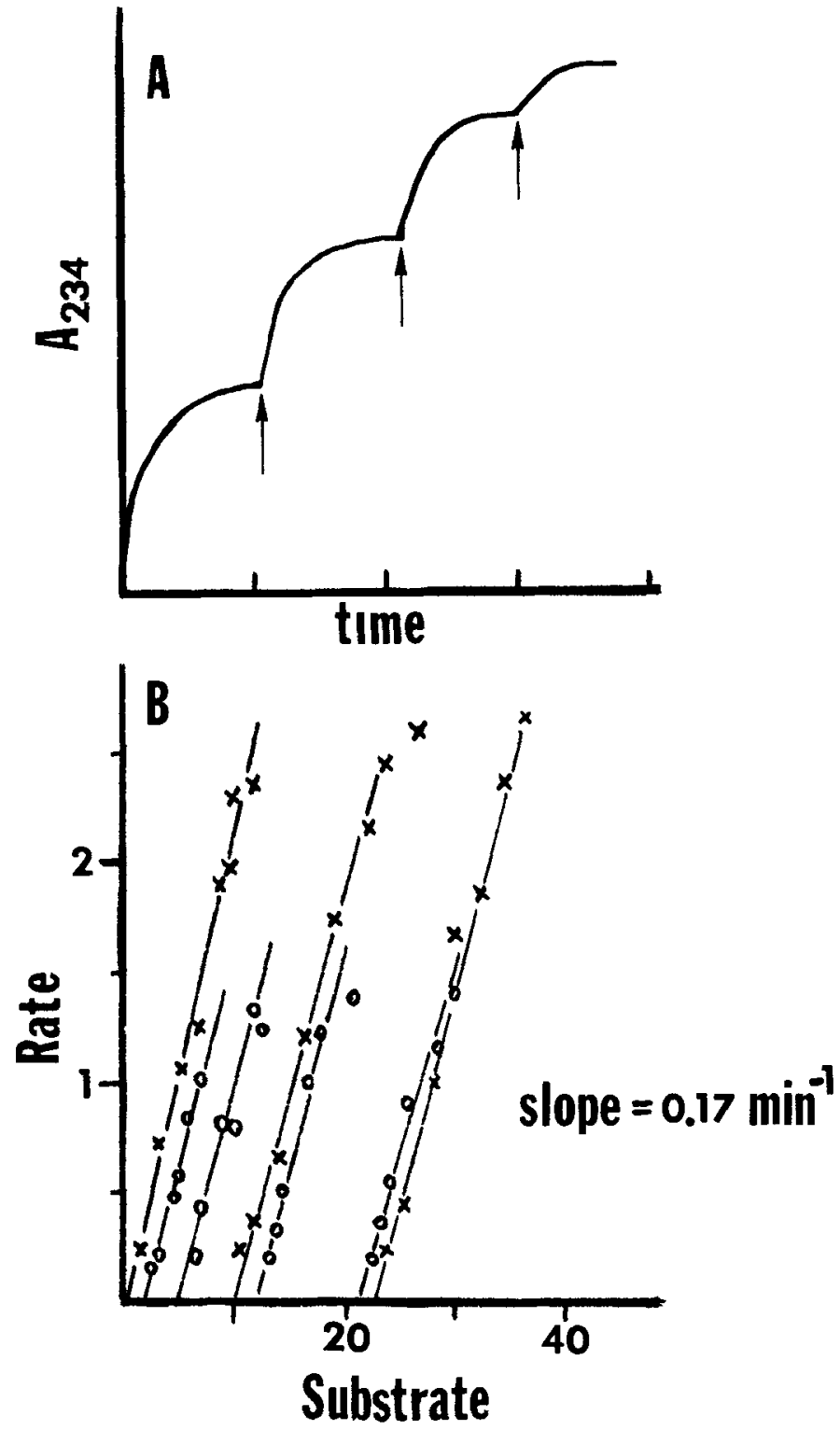

Figure 10. The self-catalyzed destruction of lipoxygenase with eicosatrienoic acid. Part $A$ indicates formation of conjugated diene with time. Additional enzyme was added, as indicated by arrows, to get complete reaction of initial substrate. Part $B$ shows the instantaneous rates during the course of reaction involving several enzyme additions. 
diminished by the presence of glutathione,but did stop immediately after adding glutathione peroxidase.

The lipoxygenase activity was slowly recovered after NEM was added to combine with the free mercaptan. The lag following NEM addition may be due in part to slow removal of mercaptan, but the definite lag after adding lipoxygenase suggests that some intermediate must accumulate prior to attainment of the maximal enzymic rate. A series of experiments convinced us that soybean lipoxygenase is activated by lipid peroxide. This was shown most clearly when the lag phase was eliminated completely by small amounts of added product. A number of conflicting reports on the lag phenomenon with lipoxygenases appear in the literature (Tappel et al,1952; Haining \& Akelrod, 1958; Koch et al, 1958; Koch,1968) but most can be reconciled with the earlier report of product activation(Haining \& Akelrod, 1958). Our results with glutathione peroxidase suggest that the product activation may be an obligatory feature of the mechanism for both systems.

In addition to the lag phase, we found another interesting feature of soybean lipoxygenase was its tendency to inactivate itself prior to complete use of added substrate. Thus,as shown in Fig.10, the rate of reaction drops to zero until fresh enzyme is added. The reaction then proceeds with no lag phase, and to an extent dependent upon the amount of enzyme added, until it stops again. The instantaneous velocities obtained at various points along the curve are replotted versus substrate concentration and show reproducibly inear decreases in velocity. The decrease in velocity in the presence of saturating levels of substrate is a measure of the decrease in active enzyme. The average slope for the experimental points gave a value of $0.17 \mathrm{~min}^{-1}$ for the destruction of lipoxygenase in the presence of $8,11,14$-eicosatrienoate and 02 . More detailed studies to be reported elsewhere indicate that the enzyme-limited extent of reaction can be interpreted most simply as self-catalyzed destruction at the stage of active enzyme-substrate- $\mathrm{O}_{2}$ complex. This phenomenon occurs to different degrees with different substrates, so each may be characterized by a different rate constant in the self-destruction reaction of lipoxygenase. The approximate values in Table 5 show a tenfold range from linoleate to docosahexaneoate.

There are some important similarities in the inability of lipoxygenase to sustain its catalytic activity under conditions necessary for reaction and the recognized lability of the vesicular gland dioxygenase (reaction 1,Fig.1). Both systems are characterized by varied specific activities between preparations and by a rapid loss of activity in reacting systems, so further reaction requires not fresh cofactors, 
Table 5. The self-catalyzed destruction of lipoxygenase in the presence of unsaturated acids.

\begin{tabular}{cc}
$\begin{array}{c}\text { Fatty Acid } \\
\text { Substrate }\end{array}$ & $\begin{array}{c}\text { Approximate } \\
k \text { Value }\end{array}$ \\
\hline $18: 2(n-6)$ & 1.2 sec -1 \\
$20: 2(n-6)$ & 1.1 \\
$18: 3(n-3)$ & 3.7 \\
$18: 3(n-6)$ & 2.9 \\
$20: 3(n-3)$ & 5.7 \\
$20: 3(n-6)$ & 3.5 \\
$20: 4(n-6)$ & 7.8 \\
$20: 5(n-3)$ & 11.4 \\
$22: 6(n-3)$ & 13.8 \\
\hline
\end{tabular}

but fresh catalyst. A number of unsaturated fatty acids that occur naturally in tissues may be oxidized but do not serve as precursors for the eventual formation of prostaglandins. It seems possible the vesicular gland dioxygenase may be inactivated irreversibly by action on those acids, as well as precursor acids. Such inactivation could diminish the capacity of the tissue to form the hormonal compounds and help emphasize the significance of the formation of new protein as a regulatory mechanism in prostaglandin synthesis.

\section{SUMMARY}

TLC of total radioactive lipids from incubation mixtures allowed quantitative estimates of the many products of the multi-enzyme complex that oxidatively rearranges fatty acids into prostaglandins. The enzymes appear together in subcellular fractions that sediment more rapidly than endoplasmic reticulum from liver. Although enzyme preparations produce $P G F / P G E$ in a ratio of 0.5 , added glutathione stimulates the internal 1,2 hydride shift(similar to that observed with glyoxalase) to increase PGE formation. On the other hand, adding $\mathrm{Cu}^{++}$plus dithiol (especially dihydrolipoamide)stops PGE formation and stimulates reduction to form PGF. The supply of metabolic intermediates for these reactions, in turn, is limited by the activity of the enzyme catalyzing the initial combination of $\mathrm{O}_{2}$ with the polyunsaturated fatty acid. This dioxygenase appears to react by a mechanism including a reducible intermediate form, and is unstable under the conditions necessary for reaction.

\section{ACKNOWLEDGMENTS}

This work was supported in part by a grant from the National Science Foundation (GB-8243) and a National Institutes of Health Training Grant(GM-00187) (William Smith).

\section{REFERENCES}

Bergstrom,s. 1967. Isolation, structure and action of the prostaglandins. In: Prostaglandins. Proc. II Nobel Symp.,S. Bergstrym \& B. Samuelsson,Eds., Almqvist \& Wiksell, Stockholm. p.2I. 
Daniels,E.G.,W.C. Krueger,F.P. Kupiecki,J.E. Pike and W.P. Schneider 1968. Isolation and characterization of a new prostaglandin isomer. J.Amer. Chem. Soc. 90:5894.

Eibl,H.,E.E. Hill and W.E.M. Lands 1969. The subcellular distribution of acyltransferases which catalyze the synthesis of phosphoglycerides. Europ.J.Biochem. 9:250.

Granstrom,E.,W.E.M. Lands and B.Samuelsson 1968. Biosynthesis of $9 \alpha, 15$-dihydroxyprost-13-enoic acid. J.Biol. Chem. 243: 4104 .

Gréen, K. and B. Samuelsson 1964. Thin-layer chromatography of the prostaglandins. J.Iipid Res. 5:117.

Haining, J.L. and B. Axelrod 1958. Induction period in the lipoxidase-catalyzed oxidation of linoleic acid and its abolition by substrate peroxide. J.Biol.Chem.232:193.

Hamberg,M. and B. Samuelsson 1967. On the specificity of the oxygenation of unsaturated fatty acids catalyzed by soybean lipoxidase. J.Biol. Chem. 242:5329.

Koch,R.B. 1968. Calcium ion activation of lipoxidase. Arch. Biochem. Biophys. 125:303.

Koch,R.B.,B. Stern and C.G. Ferrari 1958. Linoleic acid and trilinolein as substrates for soybean lipoxidase(s). Arch. Biochem. Biophys. 78:165.

Lands, W.E.M. and B. Samuelsson 1968. Phospholipid precursors of prostaglandins. Biochim. Biophys. Acta 164:426.

Lapidus,M.,N.H. Grant and H.E. Alburn 1968. Enzymatic preparation and purification of prostaglandin $\mathrm{E}_{2}$. J.Lipid Res. 9: 371 .

Little,C. and P.J. O'Brien 1968. An intracellular GSH-peroxidase with a lipid peroxide substrate. Biochem. Biophys. Res. Comm. 31: 145 .

Little,C.,R. Olinescu,K.G. Reid and P.J. O'Brien 1970. Properties and regulation of glutathione peroxidase. J.Biol. Chem. 245: 3632

Lohman, K. 1932. A study of the enzymic transformation of synthetic methylglyoxal to lactic acid. Biochem. $z$. 254:332.

O'Brien,P.J. 1969. Intracellular mechanisms for the decomposition of a lipid peroxide. I. Decomposition of a lipid peroxide by metal ions, heme compounds and nucleophiles. Canad.J.Biochem. 47:485.

Racker,E. 1951. The mechanism of action of glyoxalase. J. Biol. Chem. 190:685.

Rose,I.A. 1957. Mechanism of action of glyoxalase I. Biochim. Biophys. Acta 25:214. 
Samuelsson, B. 1967. Biosynthesis and metabolism of prostaglandins. Progr.Biochem. Pharmacol. 3: 59 .

Samuelsson,B. 1969. Biosynthesis of prostaglandins. Progr. Biochem.Pharmacol. 5: 109.

Seubold,F.H.,Jr. and W.E. Vaughan 1953. Acid-catalyzed decomposition of cumene hydroperoxide. J.Amer.Chem.Soc. 75: 3790

Sigel,H, 1969. Catalase and peroxidase activity of $\mathrm{Cu}^{++}$ complexes. Angew. Chem. Int.Ed.Engl. $\underline{8}: 167$.

Tappel,A.L.,P.D. Boyer and W.O. Lundberg 1952. The reaction mechanism of soy bean lipoxidase. J.Biol.Chem. 199:267.

van Dorp,D.A. 1967. Aspects of the biosynthesis of prostaglandins. Progr.Biochem. Pharmacol. $\underline{3}: 71$. 